\title{
Anatomy of acetone photolysis
}

A remarkable series of measurements of the photodissociation of a simple molecule points to a field in which experimentalists will have the advantage for a long time to come.

Here, one might think, is a simple problem: take a simple molecule, give it more energy than its binding-energy by means of an absorbed photon from a sufficiently energetic laser and then work out how the energy will be distributed between the fragments. On the face of things, the problem is but another version of a task in elementary mechanics that young people might be expected to solve: an object is impulsively disrupted, when what matters is that momentum is conserved. But real molecules, even simple molecules, are more complicated than that.

The immediate difficulty is that even the simplest molecular fragments are not billiard balls, but have internal degrees of freedom, rotation for example, whose excitation energy is small enough to compete effectively for a share of energy that would otherwise be shed by translation. (Nuclear physicists are often luckier, in that internal excitation of their reaction fragments can often be ignored.) It cannot be for nothing, after all, that J.C. Polanyi and his associates at the University of Toronto won a Nobel Prize in 1986 for their elegant, but emphatically empirical, techniques for identifying the excitation state of the products of molecular reactions between species whose internal motions are themselves well defined.

When will it be possible to predict these things? Not soon, it seems. Even the simpler problem of telling how quickly, and by what route, an impulse of energy will be redistributed among several internal degrees of freedom in a molecule is still up in the air, 30 years or so after people first appreciated that lasers could provide impulses of energy to predictable modes of internal motion, rotations, vibrations or even electronic excitations. Working out the excitation state of the products of molecular photolysis will require that problem to be superposed on that of following the time-course of the decomposition.

Almost any issue of the Journal of Chemical Physics (which tends to publish more than 15,000 pages of text each year) will provide a snapshot of where the problem stands, but rarely more elegantly than in a study of the photodissociation of acetone by a group from Cornell University (Trentelman, K.A. et al., J. chem. Phys.91, 7,498; 1989), itself the culmination of a long series of measurements. Acetone $\left(\mathrm{C}_{3} \mathrm{H}_{6} \mathrm{O}\right)$ is the simplest ketone, consisting simply of two methyl groups attached to the carbon atom of a carbonyl group or, alternatively, of acetic acid in which the ionizable $\mathrm{OH}$ group has been replaced by a methyl group. Hardly anything, it might be thought, could be simpler.

One complication is that there are two ways in which a sufficiently excited acetone molecule can dissociate, either into a methyl $\left(\mathrm{CH}_{3}\right)$ and an acetyl radical or into two methyl radicals and carbon monoxide. The higher the excitation energy, the more important the second reaction.

Trentelman and her colleagues have used ultraviolet pulses at $193 \mathrm{~nm}$ from an argon fluoride excimer laser to excite acetone molecules in a carrier of helium; the energy is enough to ensure that the three-fragment reaction predominates. Indeed, the excess energy to be redistributed between translational and internal motion is no less than $221 \mathrm{~kJ}$ per mole (of acetone), more than a third of the initial energy of the ultraviolet photon (the equivalent of $619 \mathrm{~kJ}$ per mole). By good luck, the ultraviolet energy is only just enough to excite acetone molecules to the energy minimum of a bound, but necessarily metastable, Rydberg state, which means that molecules embark on dissociation with virtually no vibrational energy or that they are as nearly stationary as quantum systems can be, what with all that zeropoint energy.

There is a host of questions to ask about the system, not the least of which is whether dissociation takes place in one step or two. (With less excitation energy, the end products are methyl and acetyl radicals, if only briefly.) The answer is that the two carbon bonds are broken sequentially, but that the interval between these events is on the average so much shorter than the timescales of other processes that it does not matter.

The strongest evidence for that is that no less than $28 \mathrm{~kJ}$ per mole of energy ends up as rotational energy of the $\mathrm{CO}$ fragment, more than twice as much as the rotational energy of the two methyl radicals. The simple but convincing argument is that the instantaneous disintegration of an acetone molecule, which is symmetrical about the $\mathrm{C}-\mathrm{C}$ axis, would give the $\mathrm{CO}$ fragments translational but not rotational energy, but that if one methyl radical has already left, the break-up of the remaining asymmetrical fragment is almost designed to kick the $\mathrm{CO}$ into a high rota- tion state - the most commonly occupied state has 32 rotational energy quanta. The same argument leads to the conclusion that the methyl radicals will have less rotational energy - each of them is bonded to the central carbon in a direction passing through its centre of gravity.

In reality, the bulk of the energy released (124 kJ per mole) goes into translation, inferred from measured Doppler shifts of rotational lines in the fluorescence spectrum of $\mathrm{CO}$ and, for the methyl radicals, from elegant time-of-flight measurements. (The total energy accounted for is some 15 per cent less than that calculated from the binding energy of acetone, which is within the limits of the errors.) The direct implication is that the interval between the excitation of a molecule and its dissociation cannot be long enough for energy to be equipartitioned, or even unequally redistributed, between the internal degrees of freedom of the intact molecule. That leads to the conclusion that the dissociation is an impulsive process, and in passing explains why the methyl radicals have less vibrational energy than might be expected.

The route to dissociation can also be determined from the measurements. In unexcited acetone molecules, the three skeletal carbon atoms and the oxygen lie in a plane, as do the same atoms in the state reached by absorption of a $193 \mathrm{~nm}$ photon, but there is an intermediate unbound state with a non-planar configuration. What the Cornell group says is that the excited state is first converted to the intermediate unbound state before dissociating.

The unnerving feature of this intricate and technically breathtaking study is that it has taken so much effort to reach a wellrounded picture of what is, after all, a reasonably simple molecular reaction. Elsewhere, in the same issue of $J$. chem. Phys., are essays in the direction of $a b$ initio calculations of some of the properties of such real systems - there is an attempt to show how classical calculations of the coupling between different vibrational modes of benzene would be affected by zero-point energy and a somewhat formal translation of Feynman's pathintegral calculus to the calculation of reaction rates. Taken together, they suggest this is a field in which the experimentalists will have the upper hand for some time to come.

John Maddox 